\title{
Comparative Study on Search Performance between GA and PSO for Stacking Optimization of Laminated Plates
}

\author{
Michio Innami* ${ }^{\mathrm{a} *}$ Yoshihiro Narita ${ }^{\mathrm{b}}$ \\ ${ }^{a}$ Department of Production Electronics and Information System Technology, Hokkaido Polytechnic College. Email:innami@hokkaido-pc.ac.jp

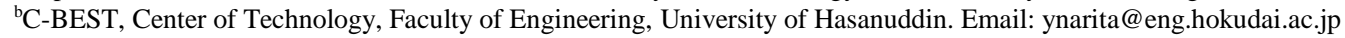

\begin{abstract}
This paper deals with the optimization problem to maximize the vibration performance of laminated composite plates by properly tailoring the fiber orientation angles in the layers. The optimization is performed by using two metaheuristic methods, Genetic Algorithm (GA) and Particle Swarm Optimization (PSO), and comparison is made to evaluate differences in search performance of the two methods. Test problems are set for the evaluation of maximizing the fundamental frequencies, and some parameters are properly tuned for efficient solution search. From the numerical experiments, it turned out that the search using PSO indicates faster convergence and better solutions than GA scheme under assumption of search domain in real number.
\end{abstract}

Keywords: Genetic algorithm; laminated composite plate; metaheuristic optimization; particle swarm optimization; vibration

\section{Introduction}

Thin structural elements made of long-fiber reinforced composite material are used widely in aerospace, automotive, marine and civil engineering applications because they may be designed to be economic in the long run due to having low mass and achieving high stiffness and strength. For such a wide range of applications, various structural models such as the analytical or numerical finite element (FE) models may be combined with optimization procedures to achieve the best design.

In the engineering optimization, there are a variety of optimization techniques, ranging from mathematical programming approaches [1] to metaheuristic approaches [2]. A class of the mathematical programming includes wellaccepted gradient-based methods, simplex algorithm for linear programming (LP), quadratic programming, dynamic programming, branch/bound method and etc. In the past few decades, however, the metaheuristic methods are more often used, such as Genetic Algorithm (GA) originally proposed by Holland [3] in 1975. Then GA was first applied to structural design in the late 1980's by Goldberg [4]. More recently, other types of nature-inspired methods have been developed, and one of the promising ones is Particle Swarm Optimization (PSO) [5-7].

The reason for increasing use of metaheuristics is mostly due to the advantages that they are simple to code and use and can provide optimum or nearly optimum solutions without falling into local solutions. The drawback is the long computation time, but the recent development of computational technology compensates the situation.

In this paper, stacking sequence problem is under consideration, where the objective function, in this case fundamental frequency, is maximized by searching the optimum fiber orientation angles in all the layers of symmetrically laminated rectangular plates. From metaheuristics, two representative methods are chosen, namely, Genetic Algorithm (GA) and Particle Swarm Optimization (PSO), and comparisons are made to evaluate the difference in search performance between the two methods. The bench mark problem is given for the evaluation of maximizing the fundamental frequencies, and the design of experiments (DoE) is used to make some search parameters being properly tuned. In the numerical experiments, it found out that the search by using PSO has shown the faster converged solutions than GA solution.

${ }^{*}$ Corresponding author. Tel.: +81-134-62-3553

3-190 Zenibako, Otaru, Japan, 047-0292 


\section{Metaheuristics and overview}

\subsection{Basic concept of $G A$}

Genetic algorithm (GA) is one of metaheuristics that are generally inspired by the process of natural selection in nature. It is within the larger class of evolutionary algorithms (EA). GA is usually used to generate nearly optimum solutions to the engineering search problem by the help of bio-inspired operators such as selection, crossover and mutation [4], and it became popular optimization approach in the past few decades. After the first works by Holland in the 1970s, he authored a book [3], and he and his co-workers have established a basic framework in GA.

In GA, a population of individuals is improved toward better solutions, where each individual is given a set of properties (genotype) to be changed and/or mutated. It is a usual practice to represent it in binary strings of 0 and 1 . The process for improvement starts from with the first generation whose members (individuals) are randomly generated. The values of fitness in all individuals are evaluated and are taken usually from the value of the objective function in the optimization. The individuals with higher fitness are selected stochastically by, for example, a roulette rule. A new generation of candidate solutions is adapted in the iteration. The iterative process is terminated based on prescribed condition, in such ways as a maximum number of generations is produced or a satisfactory fitness level is reached in the population.

The essence of GA consists in repetitive application of the selection of parents for mating and applying crossover and mutation. Particularly, the crossover and mutation are known as the most important genetic operators, and their probabilities are GA parameters needed to be tuned, called as the mutation probability and crossover probability. The population size for reasonable settings that depend on the problem being worked on.

\subsection{Basic concept of PSO}

As GA is a method inspired by principle observed in nature, a particle swarm optimization (PSO) is also one of metaheuristics and belongs to a class of evolutionary algorithm [4]. The advantages of PSO include no requirement for the gradient information in the problem and capability of searching a large space of feasible solutions. Just like GA, PSO is a numerical method to optimize in iterative process by improving individual solutions with respect to a given measure of quality. So, it requires a population of candidate solutions to be moved in the searchspace, but there is no notion like cross-over, mutation and mating of parents and that generation changes from parents to children.

The iteration process is repeated by simple mathematical formula by using the particle's position and velocity vectors. A new position is decided by adding three vectors, an inertia vector of a particle (difference of the present and past position vectors), a vector heading to the best position of an individual particle, and a vector heading to the global best among the population. Coefficients are multiplied to each vector and tuned as input parameters.

PSO was first originated by Kennedy, Eberhart and Shi [6] and was designed to simulated social behaviors of the movement in a flock of birds and school of fish. Kennedy and Eberhart published a book [7] to describe many theoretical aspects in PSO and swarm intelligence. Extensive surveys of PSO applications were made by Poli $[8,9]$. Recently, a comprehensive review on theoretical and experimental works on PSO was published by Bonyadi and Michalewicz [10].

To summarize, algorithm of PSO is to have a swarm (population) of particles (feasible solutions) and the particles are moved following the formula in the search-space according to a few simple formulae. When improved positions are being discovered, these will then come to guide the next movements of the swarm. The process is repeated until a satisfactory solution will eventually be discovered, although the convergence is not guaranteed in strict mathematical sense.

\subsection{Overview of literature on plate stacking optimization}

For optimization of mechanical behaviors in laminated composite plates, the pioneering studies of Bert [11, 12], in which the fiber-orientation angles (design variables) that maximize the fundamental frequency (objective function) of laminated plates were sought. Since these early studies, a variety of approaches have been used to identify the lay-ups that maximize the frequencies and buckling loads of laminated plates. In the early days, metaheuristic methods and supporting computational environment were not available, lamination parameters coupled with mathematical programming were used by Fukunaga and others [13]. Zhao and Narita [14] used the complex method (extension of simplex method) for the problem. Todoroki and Haftka [15] used GA with repair strategy. The progress of design and optimization on composites was summarized in a textbook [16] in 1999.

In the years of 2000's, GA and metaheuristic approaches have evolved, and Todoroki and his co-workers have published some papers [17-19]. Narita has also presented a number of related papers [20-22] by developing a layerwise optimization method, where the physics-oriented behaviors are utilized in the solution process.

More recently in the 2010's, significant progress has been observed by using GA and PSO, or even GA-PSO combined method, to solve the stacking sequence problem. Le-Manh and Lee [23] dealt with stacking sequence optimization for the maximum strength of laminated composite plates using GA. Ehsani and Rezaeepazhand [24] solved the same optimization of laminated composite grid plates for maximum buckling load using GA. Ho-Huu and others [25] optimized laminated composite plates for maximizing buckling load using improved differential evolution. 
Schaedler and Almeida [26] optimized maximum buckling load by harmony search algorithm. In 2017, Herath and others [27] used GA to optimized structural strength and laminate optimization of self-twisting composite hydrofoils. Vosoughi and co-workers $[28,29]$ presented a mixed finite element and improved GA for maximizing buckling load of stiffened laminated composite plates. These authors also made academic efforts to combine different ideas and presented maximized buckling load by using FE-GAs-PSO, a combined approach.

For comparison of GA and PSO, Zhou and others conducted a comparative study of improved GA and PSO in solving multiple traveling salesmen problem [30]. Modification from a simple GA was made by Pathan and others [31] by GA for optimizing the damping response of composite laminates. Javidrad and his colleagues [32] combined PSO with a simulated annealing (SA), and named as a hybrid PSO-SA method. Akmar and others [33] summarized recent progress in probabilistic multi-scale optimization of hybrid laminated composites. As overviewed on the applications of GA, PSO and heuristic methods above, a large number of studies are made on applications of GA and PSO to the staking sequence problem of laminated composite plates. The comparison studies of search performance between GA and PSO have not been carried out enough, and therefore in the present paper, the research on clarifying the difference in performance will be made for the purpose.

\section{Problem description for stacking sequence optimization of laminated composite plates}

Consider a laminated plate composed of orthotropic laminas as shown in Fig. 1. The stress-strain equations in the $k$-th layer can be written as

$$
\left\{\begin{array}{l}
\sigma_{x} \\
\sigma_{y} \\
\tau_{\mathrm{xy}}
\end{array}\right\}^{(k)}=\left[\begin{array}{lll}
\bar{Q}_{11} & \bar{Q}_{12} & \bar{Q}_{16} \\
\bar{Q}_{12} & \bar{Q}_{22} & \bar{Q}_{12} \\
\bar{Q}_{16} & \bar{Q}_{26} & \bar{Q}_{66}
\end{array}\right]^{(k)}\left\{\begin{array}{l}
\varepsilon_{x} \\
\varepsilon_{y} \\
\gamma_{x y}
\end{array}\right\}
$$

where $\bar{Q}_{i j}{ }^{(k)}(i, j=1,2$ and 6$)$ are the elastic constraints of the $k$-th layer. The $\bar{Q}_{i j}{ }^{(k)}$ are determined by the coordinate transformation of the Hooke's law for orthotropic plate $Q_{i j}{ }^{(k)}$ $(i, j=1,2$ and 6$)$ given by

$$
\begin{aligned}
& Q_{11}=E_{\mathrm{L}} /\left(1-v_{\mathrm{LT}} v_{\mathrm{TL}}\right), Q_{22}=E_{\mathrm{T}} /\left(1-v_{\mathrm{LT}} v_{\mathrm{TL}}\right), \\
& Q_{12}=v_{\mathrm{TL}} Q_{11}=v_{\mathrm{LT}} Q_{22} \text { and } \mathrm{Q}_{66}=G_{\mathrm{LT}}
\end{aligned}
$$

where $E_{\mathrm{L}}$ and $E_{\mathrm{T}}$ are the moduli of elasticity in the $L$ and $T$ directions, respectively, $G_{\mathrm{LT}}$ is the shear modulus, and $v_{\mathrm{LT}}$ and $v_{\mathrm{TL}}$ are the Poisson ratios, as shown in Fig. 1.
The maximum strain energy $U_{\max }$ and kinetic energy $T_{\max }$ are derived as

$$
\begin{aligned}
& U=\frac{1}{2} \iint_{A}\{\kappa\}^{T}[D]\{\kappa\} d A \\
& T=\frac{1}{2} \rho \iint_{A}\left\{\frac{\partial W}{\partial t}\right\}^{2} d A
\end{aligned}
$$

where $\{\kappa\}$ is curvature vectors defended by

$$
\{\kappa\}=\left\{-\frac{\partial^{2} W}{\partial x^{2}}-\frac{\partial^{2} W}{\partial y^{2}}-\frac{\partial^{2} W}{\partial x \partial y}\right\}^{T}
$$

$[D]$ is the bending stiffness matrix, $A$ is area of the plate, $\rho$ is material density of CFRP, $h$ is thickness of plate, and $W$ is bending deflection function. To simplify the analysis, the normalized co-ordinate system $O-\xi \eta(-1 \leq \xi, \eta \leq 1)$ is defined in equation

$$
\xi=\frac{2 x}{a}, \eta=\frac{2 y}{b} \text { and } \Omega=\omega a^{2} \sqrt{\frac{\rho}{D_{0}}}
$$

where $\xi$ and $\eta$ are dimensionless coordinates, $\Omega$ is dimensionless frequency parameter, $\omega$ is the angular frequency, and $D_{0}$ is reference plate stiffness represented by

$$
D_{0}=\frac{E_{\mathrm{T}} h^{3}}{12\left(1-v_{\mathrm{LT}} v_{\mathrm{TL}}\right)}
$$

Then, the deflection is expressed by

$$
W(\xi, \eta, t)=\sum_{m=0}^{M-1} \sum_{n=0}^{N-1} A_{m n} X_{m}(\xi) Y_{n}(\eta) \sin \omega t
$$

with

$$
\begin{aligned}
& X_{m}(\xi)=\xi^{m}(\xi+1)^{b c 1}(\xi-1)^{b c 2} \\
& Y_{n}(\eta)=\eta^{n}(\eta+1)^{b c 3}(\eta-1)^{b c 4}
\end{aligned}
$$

where $A_{m n}$ is unknown coefficients and $X_{m}(\xi)$ and $Y_{n}(\eta)$ are the displacement functions which satisfy at least the kinetic boundary conditions on the edge. The $b c i(i=1,2,3$ and 4$)$ is the boundary index which is used to satisfy the boundary conditions on each edge, and they are defined [34] as

$$
b c 1, b c 2, b c 3, b c 4= \begin{cases}0 & \text { (free) } \\ 1 & \text { (simple support }) \\ 2 & \text { (clamp) }\end{cases}
$$

The stationary value is obtained by

$$
\frac{\partial\left(U_{\max }-T_{\max }\right)}{\partial A_{m n}}=0 \quad(m n=0,1,2, \ldots,(M-1))
$$

The result of the minimization process Eq. (11) yields frequency equation in terms of unknown coefficient $A_{m n}$ as 


$$
\left|[K]-\Omega^{2}[M]\right|=0
$$

where $[K]$ is the total stiffness matrix and $[M]$ is the total mass matrix. Solving this eigenvalue equation gives the nondimensional frequency parameter $\Omega$ to be optimized.

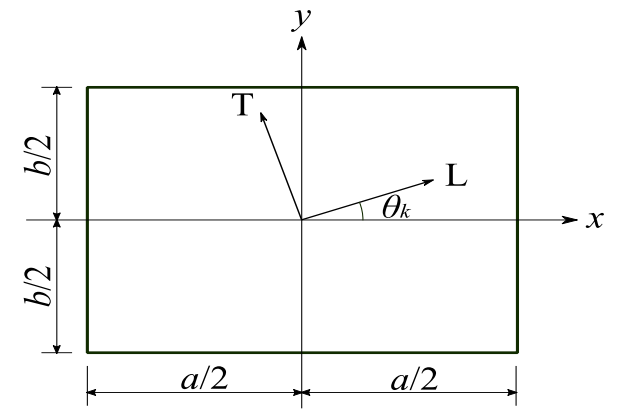

Figure 1. Laminated composite rectangular plate and coordinates

\section{Numerical experiments by GA and PSO}

\subsection{Problem setting}

This study employs GA and PSO for searching nearly optimal solutions. The design variables, i.e., the fiber orientation angles of laminas are treated as continuous values. An 8-layer symmetric CFRP laminated plate shown in Fig. 1 is analyzed. The dimensions and material constants are as follows:

$$
a=b=150 \mathrm{~mm} . h=15 \mathrm{~mm}
$$

(Note that the frequency parameter is normalized with $h$, and the value of $a / h$ does not affect it)

$$
\begin{aligned}
& E_{L}=138 \mathrm{GPa}, E_{T}=8.96 \mathrm{GPa}, G_{L T}=7.1 \mathrm{GPa} \\
& v_{L T}=0.3, \rho=1578 \mathrm{~kg} / \mathrm{m}^{3}
\end{aligned}
$$

This study tries to find the fiber orientation angles of the laminas that composes the plate, which make the fundamental natural frequency maximum.

The boundary conditions are assumed as CSFF, SSSS, CCFF, CSSS, where the sequence of four characters stands for the type of constraint on the left, lower, right, and upper edge, starting from the left edge. The letters F, S, and C denote Free, Simply supported, and Clamped, respectively.

\section{2. $G A$}

Continuous values are to be stored in the genes on a chromosome. Roulette wheel selection is adopted. Linear scaling is applied so that the selection might be operated effectively. It scales the fitness values using the following equations

$$
\begin{aligned}
f_{s}= & a f+b \\
a & =\frac{(c-1) f_{\text {avg }}}{f_{\max }-f_{\text {avg }}} \quad b=\frac{f_{\text {avg }}\left(f_{\text {msx }}-c f_{\text {avg }}\right)}{f_{\max }-f_{\text {avg }}}
\end{aligned}
$$

where $f_{\max }$ and $f_{\text {avg }}$ are the maximum and average values of the original function, respectively. In this study, the constant $c$ is set to 2 . The elite strategy is also applied. This study adopts two-point crossover and mutation operators.

\subsection{PSO}

The position and velocity vectors of each particle in a swarm are defined as follows.

$$
\begin{gathered}
\boldsymbol{v}_{i}^{k+1}=w \boldsymbol{v}_{i}^{k}+c_{1} r_{1}\left(\text { pbest }_{i}-\boldsymbol{x}_{i}^{k}\right) \\
+c_{2} r_{2}\left(\text { gbest }-\boldsymbol{x}_{i}^{k}\right) \\
\boldsymbol{x}_{i}^{k+1}=\boldsymbol{x}_{i}^{k}+\boldsymbol{v}_{i}^{k+1}
\end{gathered}
$$

where $\boldsymbol{x}_{i}^{k}$ and $\boldsymbol{v}_{i}^{k}$ are $n$-dimensional position and velocity vectors of particle $i$ at the $k$-th generation, respectively. pbest $_{i}$ and gbest are the position vectors where the objective function took the best value so far in the track of particle $i$, and of the whole particles, respectively. $w, c_{1}$, and $c_{2}$ are constants, $r_{1}$, and $r_{2}$ are uniform random numbers between 0 and 1 .

\section{Parameter setting}

The parameters of GA and PSO should be selected properly. This study employs DoE approach for determining them [35]. In both GA and PSO, four factors are chosen, then 4 levels are set for each of them. Normally there are $4^{4}$ combinations of parameters. However with DoE approach, the number of experiments can be extremely reduced. The experiments for determining the parameters are planned to be executed according to the orthogonal array $\mathrm{L}_{16}\left(4^{4}\right)$ as shown in Table 1. It requires 16 types of experiments.

Table 1. Orthogonal array $\mathrm{L}_{16}\left(4^{4}\right)$

\begin{tabular}{ccccc}
\hline \multirow{2}{*}{ Experiment } & \multicolumn{4}{c}{ Level } \\
\cline { 2 - 5 } & A & B & C & D \\
\hline 1 & 1 & 1 & 1 & 1 \\
2 & 1 & 2 & 2 & 2 \\
3 & 1 & 3 & 3 & 3 \\
4 & 1 & 4 & 4 & 4 \\
5 & 2 & 1 & 2 & 3 \\
6 & 2 & 2 & 1 & 4 \\
7 & 2 & 3 & 4 & 1 \\
8 & 2 & 4 & 3 & 2 \\
9 & 3 & 1 & 3 & 4 \\
10 & 3 & 2 & 4 & 3 \\
11 & 3 & 3 & 1 & 2 \\
12 & 3 & 4 & 2 & 1 \\
13 & 4 & 1 & 4 & 2 \\
14 & 4 & 2 & 3 & 1 \\
15 & 4 & 3 & 2 & 4 \\
16 & 4 & 4 & 1 & 3 \\
\hline
\end{tabular}




\subsection{Parameters of $G A$}

Population size, elite count, crossover rate, and mutation rate are defined as factors of DoE. Table 2 shows the factors and their levels. The maximum number of generations are chosen as the products of the population size and itself might be equal among all the levels.

\subsection{Parameters of PSO}

Population size, $w, c_{1}$, and $c_{2}$ are defined as factors of DoE. The factors and their levels are shown in Table 3. The maximum number of generations are chosen as the products of the population size might be equal among all the levels, same as the case of GA.

\section{Experimental results}

In accordance with the experimental plan in Table 1, every experiment was conducted 3 times. Tables 4 and 5 present the fundamental frequencies derived from above trials by GA and PSO, respectively. Figures 2 and 3 show the values with each factor level. Consequently, the parameters of GA and PSO were selected as shown in Table 6 and 7, respectively. The maximum number of generation is extended to 500 in both cases.

Table 2. Factors and their levels of GA parameters

\begin{tabular}{|c|c|c|c|c|c|}
\hline & \multirow{2}{*}{ Factor } & \multicolumn{4}{|c|}{ Level } \\
\hline & & 1 & 2 & 3 & 4 \\
\hline \multirow{2}{*}{ A } & $\begin{array}{l}\text { Population } \\
\text { size }\end{array}$ & 20 & 40 & 80 & 160 \\
\hline & $\begin{array}{l}\text { Max. } \\
\text { generation }\end{array}$ & 200 & 100 & 50 & 25 \\
\hline $\mathrm{B}$ & Elite count & 1 & 3 & 5 & 10 \\
\hline $\mathrm{C}$ & $\begin{array}{l}\text { Crossover } \\
\text { rate }\end{array}$ & 0.3 & 0.5 & 0.7 & 0.9 \\
\hline D & $\begin{array}{l}\text { Mutation } \\
\text { rate }\end{array}$ & 0.01 & 0.03 & 0.05 & 0.10 \\
\hline
\end{tabular}

Table 3. Factors and their levels of PSO parameters

\begin{tabular}{llcccc}
\hline \multirow{2}{*}{ Factor } & \multicolumn{4}{c}{ Level } \\
\cline { 2 - 6 } & 1 & 2 & 3 & 4 \\
\hline & $\begin{array}{l}\text { Population } \\
\text { size }\end{array}$ & 20 & 40 & 80 & 160 \\
\hdashline $\mathrm{A}$ & $\begin{array}{l}\text { Max. } \\
\text { generation }\end{array}$ & 200 & 100 & 50 & 25 \\
\hline $\mathrm{B}$ & $w$ & 0.3 & .05 & 0.7 & 0.9 \\
\hline $\mathrm{C}$ & $c_{1}$ & 1.0 & 1.5 & 2.0 & 2.5 \\
\hline $\mathrm{D}$ & $\mathrm{c}_{2}$ & 1.0 & 1.5 & 2.0 & 2.5 \\
\hline
\end{tabular}

With above parameters, the search experiments were carried out 3 times by each method. The calculation was executed under Java environment 1.8.0 using a laptop (Core 2 Duo, T9300, 2.50GHz, 4GB RAM, Windows 10 64-bit).
The results are shown in Table 8. "Converged" means the point when $\Omega_{1}$ reaches the final value.

The transition of $\Omega_{1}$ in every trial is shown in Figs. 4-7. It turns out that the calculation time per generation is almost same between GA and PSO, although the final $\Omega_{1}$ values are slightly better derived from PSO than from GA. Furthermore the search with PSO converges faster than those of GA. The results indicate that the calculation is sufficient at least up to $40^{\text {th }}$ generation. In contrast, with GA, the generation considered sufficient for convergence cannot be estimated within $500^{\text {th }}$ generation.

\section{Conclusions}

Search performance for optimizing the natural frequencies of fundamental mode was compared between GA with PSO. Appropriate parameters of GA and PSO were selected by DoE approach. It revealed the performance with PSO is better than with GA. In maximization, higher values of $\Omega_{1}$ were obtained by using PSO. Moreover, the results showed that all the solutions are converged until at least $40^{\text {th }}$ generation.

\section{References}

[1] R.T. Haftka, Z. Gürdal, Elements of Structural Optimization (Solid Mechanics and Its Applications, Springer, 1991.

[2] K.L. Du, M.N.S. Swamy, Search and Optimization by Metaheuristics: Techniques and Algorithms Inspired by Nature, Birkhaeuser, 2016.

[3] J.H. Holland, Adaptation in Natural and Artificial Systems: An Introductory Analysis with Applications to Biology, Control, and Artificial Intelligence, A Bradford Book, 1975 \& 1992.

[4] D.E. Goldberg, Genetic Algorithms in Search, Optimization, and Machine Learning, Addison-Wesley Professional, 1989.

[5] A. E. Olsson, Particle Swarm Optimization: Theory, Techniques and Applications, Nova Science Pub Inc., 2011.

[6] J. Kennedy, R. Eberhart, Particle Swarm Optimization, Proceedings of IEEE International Conference on Neural Networks. IV., 1995, pp.1942-1948.

[7] J. Kennedy, R.C. Eberhart, Swarm Intelligence. Morgan Kaufmann, 2001.

[8] R. Poli, An analysis of publications on particle swarm optimisation applications, Technical Report CSM-469. Department of Computer Science, University of Essex, UK, 2007.

[9] R. Poli, Analysis of the publications on the applications of particle swarm optimization, Journal of Artificial Evolution and Applications, 2008, pp.1-10.

[10] M.R. Bonyadi, Z. Michalewicz, Z., Particle swarm optimization for single objective continuous space problems: a review. Evolutionary Computation, 25, 2017, pp. 1-54.

[11] C.W. Bert, Optimal design of a composite material plate to maximize its fundamental frequency, Journal of Sound \& Vibration, 50, 1977, pp.229-237.

[12] C.W. Bert, Design of clamped composite material plates to maximize fundamental frequency, Journal of Mechanical Design, 1978, pp.274278

[13] H. Fukunaga, H. Sekine, M. Sato, Optimal design of symmetrically laminated plates for the fundamental frequency, Journal of Sound \& Vibration, 171, 1994, pp.219-229.

[14] X. Zhao, Y. Narita, Maximization of fundamental frequency for generally laminated rectangular plates by the complex method, Transactions of JSME 63C, 1997, pp.364-370 (in Japanese). 
Table 4. Experimental results (GA)

\begin{tabular}{ccccccccc}
\hline \multirow{2}{*}{ Experiment } & \multicolumn{9}{c}{ Level } & \multicolumn{5}{c}{ Results } \\
\cline { 2 - 8 } & A & B & C & D & Trial 1 & Trial 2 & Trial 3 & Mean \\
\hline 1 & 1 & 1 & 1 & 1 & 16.363 & 16.292 & 16.340 & 16.332 \\
2 & 1 & 2 & 2 & 2 & 16.359 & 16.361 & 16.388 & 16.369 \\
3 & 1 & 3 & 3 & 3 & 16.389 & 16.295 & 16.327 & 16.337 \\
4 & 1 & 4 & 4 & 4 & 16.371 & 16.349 & 16.285 & 16.335 \\
5 & 2 & 1 & 2 & 3 & 16.342 & 16.376 & 16.379 & 16.366 \\
6 & 2 & 2 & 1 & 4 & 16.316 & 16.359 & 16.357 & 16.344 \\
7 & 2 & 3 & 4 & 1 & 16.401 & 16.412 & 16.330 & 16.381 \\
8 & 2 & 4 & 3 & 2 & 16.319 & 16.242 & 16.259 & 16.273 \\
9 & 3 & 1 & 3 & 4 & 16.371 & 16.298 & 16.315 & 16.328 \\
10 & 3 & 2 & 4 & 3 & 16.406 & 16.355 & 16.339 & 16.367 \\
11 & 3 & 3 & 1 & 2 & 16.319 & 16.366 & 16.366 & 16.350 \\
12 & 3 & 4 & 2 & 1 & 16.353 & 16.369 & 16.363 & 16.362 \\
13 & 4 & 1 & 4 & 2 & 16.394 & 16.335 & 16.384 & 16.371 \\
14 & 4 & 2 & 3 & 1 & 16.396 & 16.371 & 16.360 & 16.376 \\
15 & 4 & 3 & 2 & 4 & 16.333 & 16.385 & 16.352 & 16.357 \\
16 & 4 & 4 & 1 & 3 & 16.278 & 16.325 & 16.295 & 16.299 \\
\hline
\end{tabular}

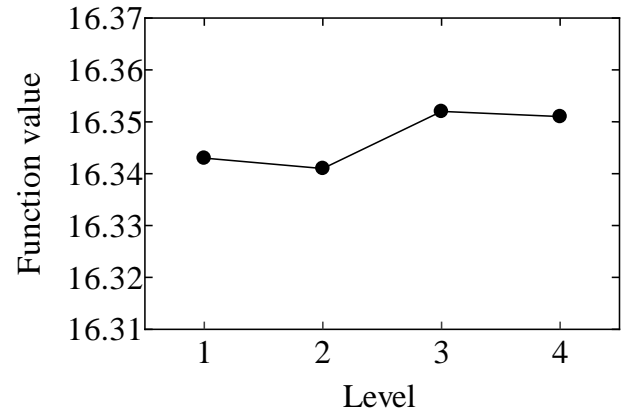

(d) Factor $\mathrm{A}$

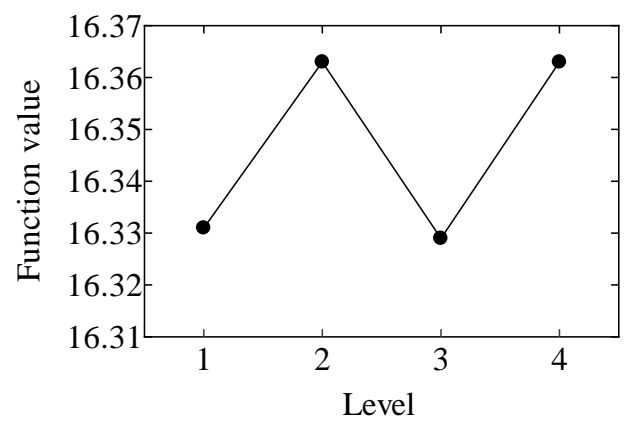

(b) Factor $\mathrm{C}$

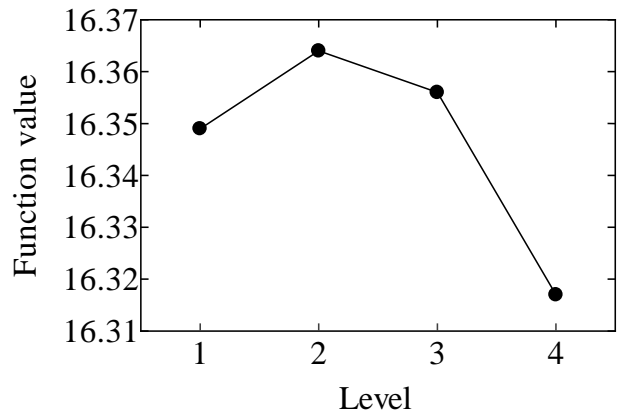

(c) Factor B

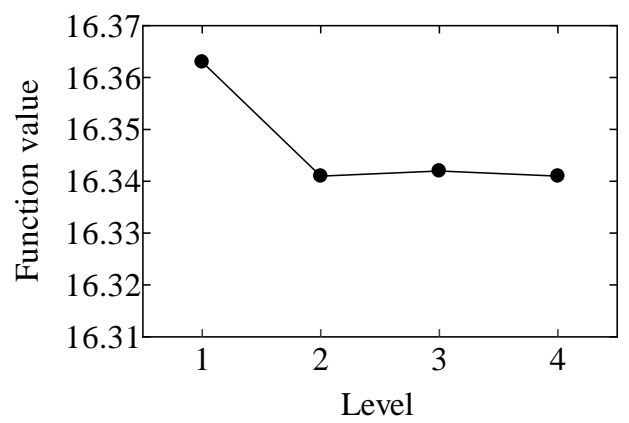

(a) Factor D

Figure 2. Main effects plot (GA) 
Table 5. Experimental results (PSO)

\begin{tabular}{ccccccccc}
\hline \multirow{2}{*}{ Experiment } & \multicolumn{3}{c}{ Level } & \multicolumn{5}{c}{ Results } \\
\cline { 2 - 8 } & A & B & C & D & Trial 1 & Trial 2 & Trial 3 & Mean \\
\hline 1 & 1 & 1 & 1 & 1 & 16.410 & 16.308 & 16.410 & 16.376 \\
2 & 1 & 2 & 2 & 2 & 16.415 & 16.415 & 16.415 & 16.415 \\
3 & 1 & 3 & 3 & 3 & 16.344 & 16.331 & 16.368 & 16.348 \\
4 & 1 & 4 & 4 & 4 & 16.278 & 16.242 & 16.187 & 16.236 \\
5 & 2 & 1 & 2 & 3 & 16.415 & 16.415 & 16.415 & 16.415 \\
6 & 2 & 2 & 1 & 4 & 16.415 & 16.415 & 16.415 & 16.415 \\
7 & 2 & 3 & 4 & 1 & 16.381 & 16.307 & 16.375 & 16.354 \\
8 & 2 & 4 & 3 & 2 & 16.338 & 16.269 & 16.301 & 16.303 \\
9 & 3 & 1 & 3 & 4 & 16.393 & 16.404 & 16.314 & 16.370 \\
10 & 3 & 2 & 4 & 3 & 16.326 & 16.393 & 16.314 & 16.344 \\
11 & 3 & 3 & 1 & 2 & 16.413 & 16.412 & 16.411 & 16.412 \\
12 & 3 & 4 & 2 & 1 & 16.291 & 16.332 & 16.363 & 16.329 \\
13 & 4 & 1 & 4 & 2 & 16.376 & 16.402 & 16.378 & 16.385 \\
14 & 4 & 2 & 3 & 1 & 16.389 & 16.336 & 16.359 & 16.361 \\
15 & 4 & 3 & 2 & 4 & 16.310 & 16.364 & 16.389 & 16.354 \\
16 & 4 & 4 & 1 & 3 & 16.329 & 16.373 & 16.367 & 16.356 \\
\hline
\end{tabular}

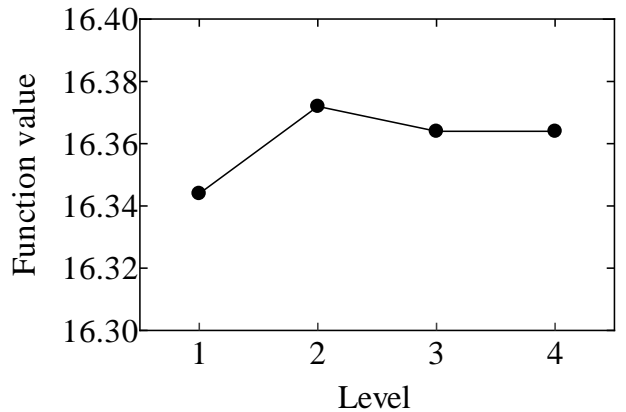

(a) Factor $\mathrm{A}$

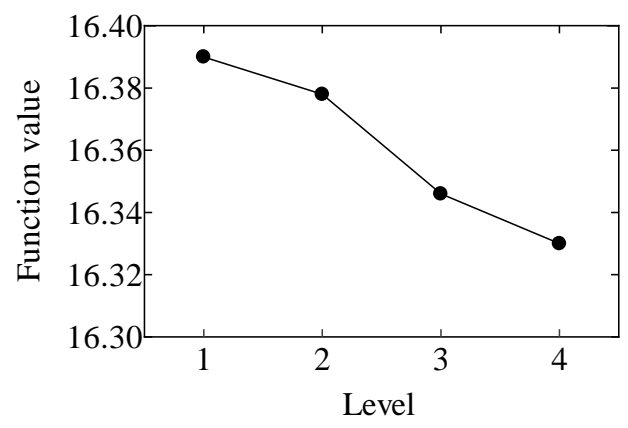

(c) Factor $\mathrm{C}$

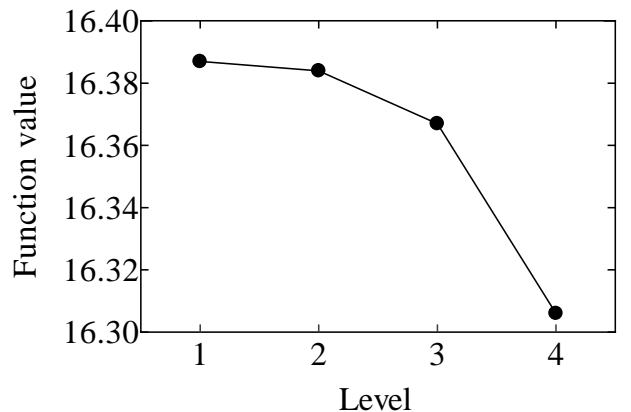

(b) Factor $\mathrm{B}$

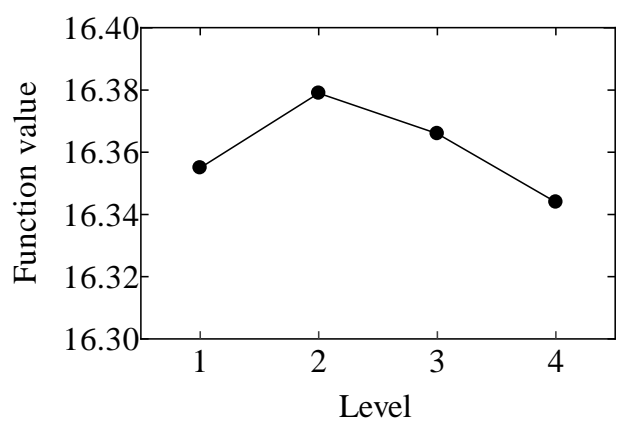

(d) Factor D

Figure 3. Main effects plot (PSO) 
Table 6. Selected parameters of GA

\begin{tabular}{ccccc}
\hline $\begin{array}{l}\text { Population } \\
\text { Size }\end{array}$ & $\begin{array}{l}\text { Maximum } \\
\text { generation }\end{array}$ & $\begin{array}{l}\text { Elite } \\
\text { count }\end{array}$ & $\begin{array}{l}\text { Crossover } \\
\text { rate }\end{array}$ & $\begin{array}{l}\text { Mutation } \\
\text { rate }\end{array}$ \\
\hline 80 & 500 & 3 & 0.5 & 0.01 \\
\hline
\end{tabular}

Table 7. Selected parameters of PSO

\begin{tabular}{lcccc}
\hline $\begin{array}{l}\text { Population } \\
\text { Size }\end{array}$ & $\begin{array}{l}\text { Maximum } \\
\text { generation }\end{array}$ & $\begin{array}{l}\text { Elite } \\
\text { count }\end{array}$ & $\begin{array}{l}\text { Crossover } \\
\text { rate }\end{array}$ & $\begin{array}{l}\text { Mutation } \\
\text { rate }\end{array}$ \\
\hline 80 & 500 & 3 & 0.5 & 0.01
\end{tabular}

Table 8. Maximized $\Omega_{1}$, stacking sequence, and elapsed time

(a) $\mathrm{CSFF}$

\begin{tabular}{|c|c|c|c|c|c|c|c|c|c|c|}
\hline & \multirow{2}{*}{ Trial } & \multicolumn{4}{|c|}{ Final $\left(500^{\text {th }}\right.$ generation $)$} & \multicolumn{4}{|c|}{ Converged } & \multirow{2}{*}{ Stacking sequence } \\
\hline & & $\Omega_{1}$ & (Mean) & Time(s) & (Mean) & Generation & (Mean) & Time(s) & (Mean) & \\
\hline \multirow{3}{*}{ GA } & 1 & 16.399 & & 16898 & & 488 & & 16494 & & {$[21.1 /-45.4 / 18.7 /-41.7] \mathrm{s}$} \\
\hline & 2 & 16.403 & $(16.401)$ & 16261 & $(16367)$ & 186 & (260) & 6079 & $(8671)$ & {$[22.6 /-42.5 / 17.5 / 19.3] \mathrm{s}$} \\
\hline & 3 & 16.402 & & 15941 & & 107 & & 3439 & & {$[21.2 /-45.9 / 20.3 / 5.6] \mathrm{s}$} \\
\hline \multirow{3}{*}{ PSO } & 1 & 16.410 & \multirow{3}{*}{$(16.413)$} & 16757 & & 20 & \multirow{3}{*}{ (20) } & 705 & \multirow{3}{*}{$(722)$} & {$[21.9 /-44.0 / 21.9 /-44.0] \mathrm{s}$} \\
\hline & 2 & 16.415 & & 17236 & $(16877)$ & 22 & & 794 & & {$[21.5 /-44.4 / 21.5 / 21.5] \mathrm{s}$} \\
\hline & 3 & 16.415 & & 16638 & & 19 & & 668 & & {$[21.5 /-44.4 / 21.5 / 21.5]_{\mathrm{s}}$} \\
\hline
\end{tabular}

(b) SSSS

\begin{tabular}{|c|c|c|c|c|c|c|c|c|c|c|}
\hline & \multirow{2}{*}{ Trial } & \multicolumn{4}{|c|}{ Final $\left(500^{\text {th }}\right.$ generation $)$} & \multicolumn{4}{|c|}{ Converged } & \multirow{2}{*}{ Stacking sequence } \\
\hline & & $\Omega_{1}$ & (Mean) & Time(s) & (Mean) & Generation & (Mean) & Time(s) & (Mean) & \\
\hline \multirow{3}{*}{ GA } & 1 & 56.290 & & 35198 & & 379 & & 26698 & & {$[-43.8 / 44.1 / 42.5 / 49.3]_{\mathrm{s}}$} \\
\hline & 2 & 56.224 & $(56.232)$ & 35266 & $(35182)$ & 61 & (225) & 4371 & (15887) & {$[48.1 /-43.9 /-42.7 /-50.5] \mathrm{s}$} \\
\hline & 3 & 56.182 & & 35083 & & 236 & & 16592 & & {$[45.4 /-45.7 /-40.4 /-22.6] \mathrm{s}$} \\
\hline \multirow{3}{*}{ PSO } & 1 & 56.319 & \multirow{3}{*}{$(56.319)$} & 36084 & & 34 & \multirow{3}{*}{ (35) } & 2570 & \multirow{3}{*}{$(2605)$} & {$[-45.0 / 45.0 / 45.0 / 45.0]_{\mathrm{s}}$} \\
\hline & 2 & 56.319 & & 35331 & $(35513)$ & 37 & & 2790 & & {$[-45.0 / 45.0 / 45.0 / 45.0] \mathrm{s}$} \\
\hline & 3 & 56.319 & & 35125 & & 34 & & 2456 & & {$[45.0 /-45.0 /-45.0 /-45.0]_{\mathrm{s}}$} \\
\hline
\end{tabular}

(c) $\mathrm{CCFF}$

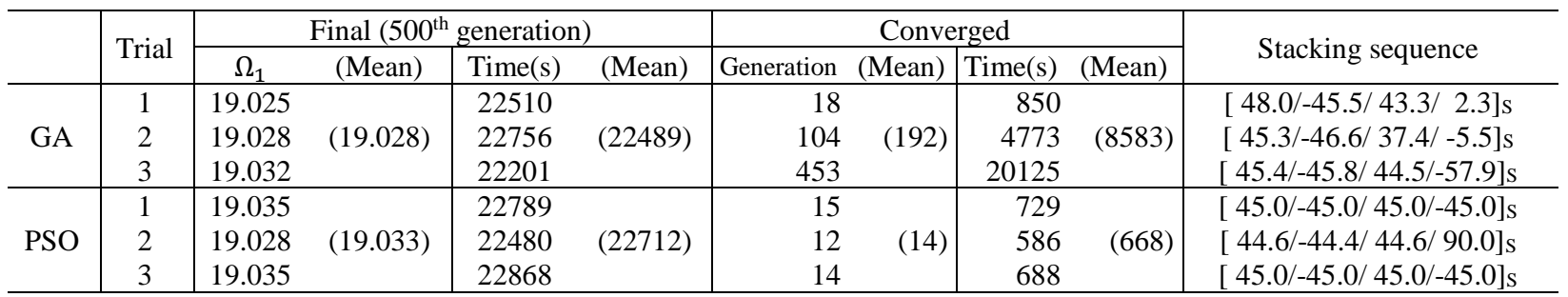

(d) CSSS

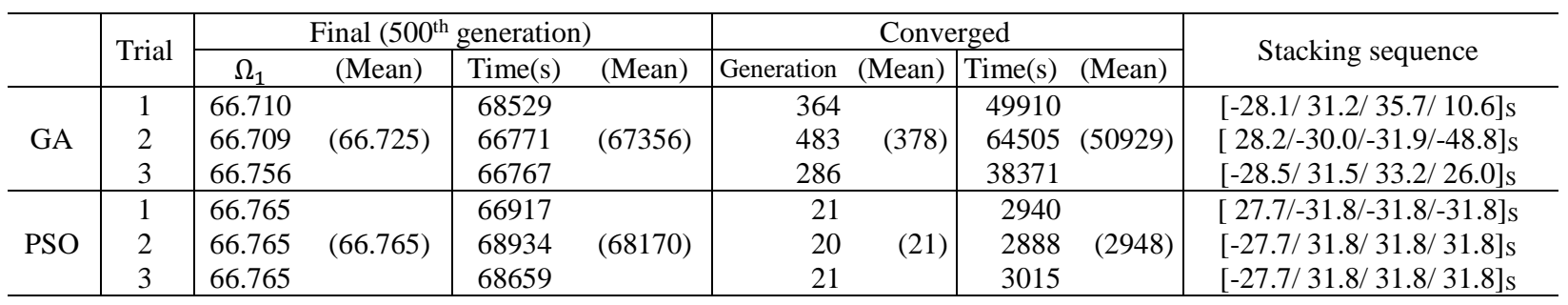




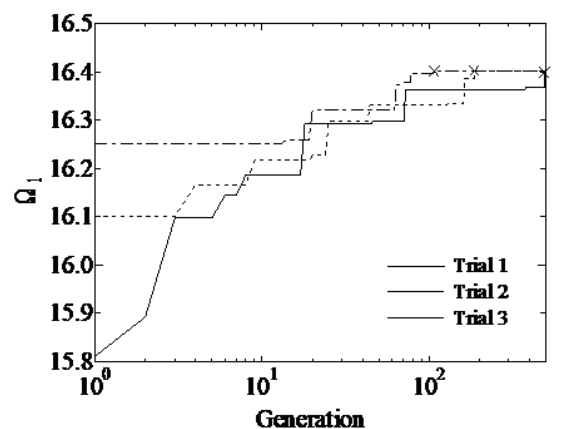

(a) GA

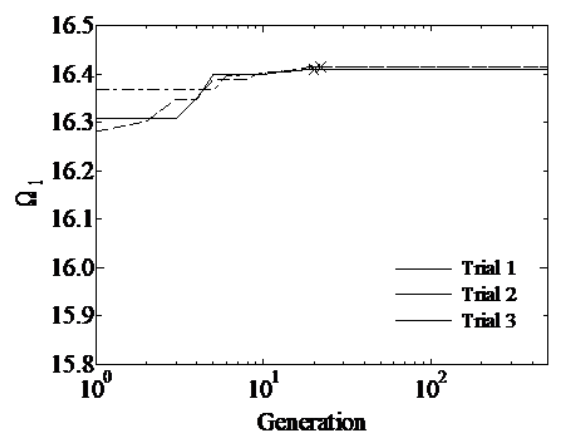

(b) PSO

Figure 4. Transition of $\Omega_{1}$ with generation for CSFF

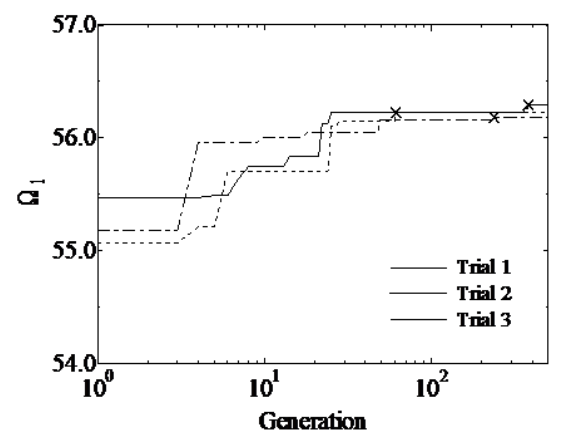

(a) GA

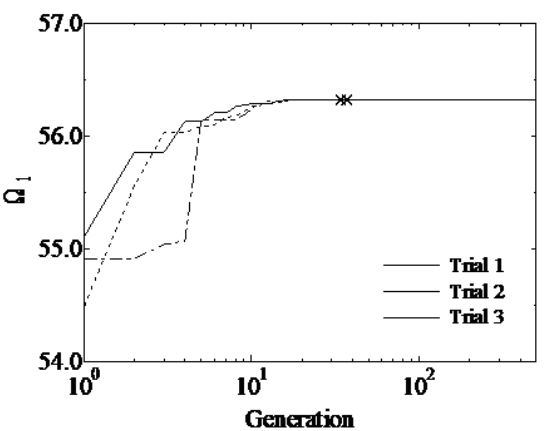

(b) PSO

Figure 5. Transition of $\Omega_{1}$ with generation for SSSS

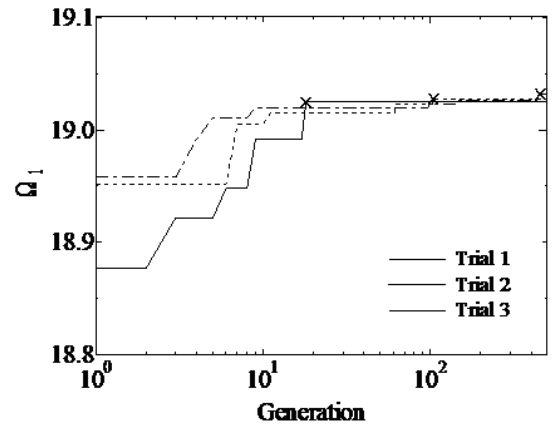

(a) GA

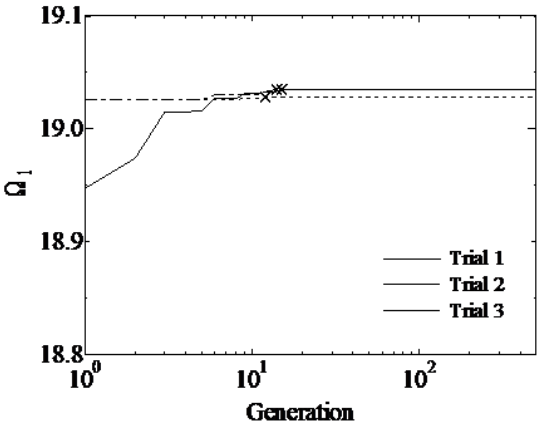

(b) PSO

Figure 6. Transition of $\Omega_{1}$ with generation for CCFF

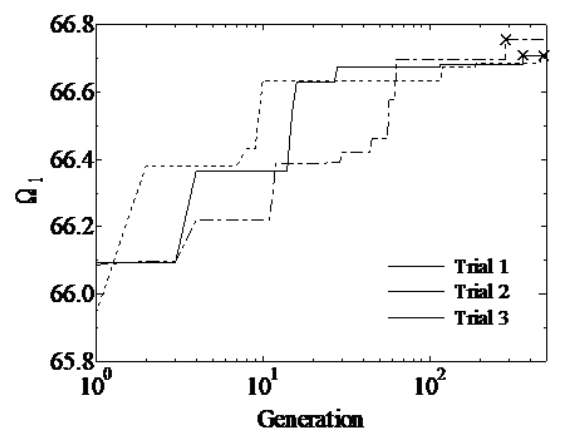

(b) GA

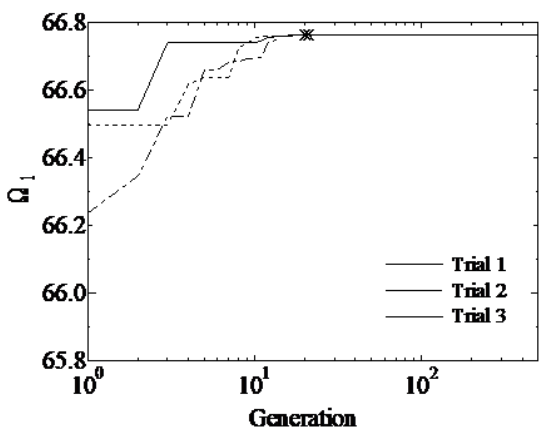

(c) PSO

Figure 7. Transition of $\Omega_{1}$ with generation for CSSS 
[15] A. Todoroki, R.T. Haftka, Stacking sequence optimization by a genetic algorithm with a new recessive gene like repair strategy, Composites Part B, 29, 1998, pp.277-285

[16] Z. Gürdal, R.T. Haftka, P. Hajela, Design and Optimization of Laminated Composite Materials, Wiley-Interscience, 1999.

[17] A. Todoroki, T. Ishikawa, Design of experiments for stacking sequence optimizations with genetic algorithm using response surface approximation, Composite Structures, 64, 2004, pp.349-357.

[18] R. Matsuzaki, A. Todoroki, Stacking-sequence optimization using fractal branch-and-bound method for unsymmetrical laminates, Composite Structures, 78, 2007, pp.537-550.

[19] A. Todoroki, M. Sekishiro, Stacking sequence optimization to maximize the buckling load of blade-stiffened panels with strength constraints using the iterative fractal branch and bound method, Composites Part B, 39, 2008, pp.842-850.

[20] Y. Narita, Layerwise optimization for the maximum fundamental frequency of laminated composite plate, Journal of Sound \& Vibration, 263, 2003, pp.1005-1016.

[21] Y. Narita, G.J. Turvey, Maximizing the buckling loads of symmetrically laminated composite plates using a layerwise optimization approach, Journal Mechanical Engineering Science, 218, 2004, pp.681-691.

[22] Y. Narita, Maximum frequency design of laminated plates w ith mixed boundary conditions, International Journal of solids \& Structures, 43, 2006, pp.4342-4356.

[23] T. Le-Manh, J. Lee, Stacking sequence optimization for maximum strengths of laminated composite plates using genetic algorithm and isogeometric analysis, Composite Structures, 116, 2014, pp.357-363.

[24] Ehsani, J. Rezaeepazhand, Stacking sequence optimization of laminated composite grid plates for maximum buckling load using genetic algorithm, International Journal of Mechanical Sciences, 119, 2016, pp.97-106.

[25] V. Ho-Huu, T.D. Do-Thi, H. Dang-Trung, T. Vo-Duy, T. NguyenThoi, Optimization of laminated composite plates for maximizing buckling load using improved differential evolution and smoothed finite element method, Composite Structures, 146, 2016, pp.132-147.

[26] F. Schaedler, de Almeida, Stacking sequence optimization for maximum buckling load of composite plates using harmony search algorithm, Composite Structures, 143, 2016, pp.287-299.

[27] M.T. Herath, B.G. Prusty, A.W. Phillips, N.S. John, Structural strength and laminate optimization of self-twisting composite hydrofoils using a Genetic Algorithm, Composite Structures, 176, 2017, pp.359-378.

[28] A.R. Vosoughi, A. Darabi, N. Anjabin, U. Topal, A mixed finite element and improved genetic algorithm method for maximizing buckling load of stiffened laminated composite plates, Aerospace Science and Technology, 70, 2017, pp.378-387.

[29] A.R. Vosoughi, A. Darabi, H.Dehghani, Forkhorji, Optimum stacking sequences of thick laminated composite plates for maximizing buckling load using FE-GAs-PSO, Composite Structures, 159, 2017, pp.361367.

[30] H. Zhou, M. Song, W. Pedrycz, A comparative study of improved GA and PSO in solving multiple traveling salesmen problem, Applied Soft Computing, 64, 2018, pp.564-580.

[31] M.V. Pathan, S. Patsias, V.L. Tagariellia, A real-coded genetic algorithm for optimizing the damping response of composite laminates, Computers \& Structures, 198, 2018, pp.51-60.

[32] F. Javidrad, M. Nazari, H.R. Javidrad, Optimum stacking sequence design of laminates using a hybrid PSO-SA method, Composite Structures, 185, 2018, pp.607-618.

[33] A.B.I. Akmar, O. Kramer, T. Rabczuk, Probabilistic multi-scale optimization of hybrid laminated composites, Composite Structures, 184, 2018, pp.1111-1125.

[34] Y. Narita, Series and Ritz-type buckling analysis, in: G.J. Turvey, I.H. Marshall (Eds.), Buckling and Postbuckling of Composite Plates, Chapman \& Hall, London, pp. 33-57 (Chapter 2), 1995.

[35] Son D, Kazem A, Romeo M. Maximising Performance of Genetic Algorithm Solver in Matlab. Engineering Letters, 24; 2016. pp. 75-83. 